\title{
INDUSTRIA DEL CEMENTO EN MÉXICO: ANÁLISIS DE SUS DETERMINANTES
}

\author{
Belem Vásquez y Salvador Corrales ${ }^{1}$
}

Fecha de recepción: 29 de abril de 2016. Fecha de aceptación: 04 de octubre de 2016.

\begin{abstract}
RESUMEN
A continuación se presenta el análisis e identificación de algunos determinantes que explican la producción de la industria del cemento en México. El objetivo del estudio fue identificar el efecto de las exportaciones y la competencia externa sobre la producción cementera, así como determinantes internos como: la inversión, el empleo y la actividad económica general. Con una función de producción aumentada se estimaron modelos de corrección de errores. Los resultados indican que la tasa de cambio de la producción de cemento se relaciona positivamente con las exportaciones y negativamente con la competencia externa. La actividad económica general mostró un fuerte efecto sobre la producción. Bajo el supuesto de que la industria opera en oligopolio, los resultados implican que no existen incentivos para favorecer la entrada de competidores.
\end{abstract}

Palabras clave: industria del cemento, producción, empresas, mercado, inversión, empleo.

Clasificación JEL: F12, F14, L13, O12, O14.

\section{The Cement Industry in Mexico: An Analysis of its Determinants}

\begin{abstract}
Below, this paper analyzes and identifies some of the determinants that explain cement industry production in Mexico. The objective of this study was to identify the effect of exports and external competition on cement production, as well as internal drivers, such as investment, employment, and overall economic activity. As a function of augmented production, error correction models were estimated. The results indicate that the cement production exchange rate is positively related with exports and negatively with external competition. Overall economic activity showed a strong effect on production. Assuming that the industry operates as an oligopoly, the results imply that there are no incentives to favor the entrance of competitors.
\end{abstract}

Key Words: Cement industry, production, companies, market, investment, employment.

1 El Colegio de la Frontera Norte, México. Correos electrónicos: belem@colef.mx y corrales@colef.mx, respectivamente. 


\section{INDUSTRIE DU CIMENTA U MEXIQUE: ANALYSE DES DÉTERMINANTS Résumé}

Ce texte presente une analyse et identification de certains déterminants qui expliquent la production de l'industrie du ciment au Mexique. L'objectif de cette étude visait à identifier l'effet des exportations et la concurrence étrangère sur la production de ciment et aussi bien que les déterminants internes tels que l'investissement, l'emploi et l'activité économique globale. En utilisant d'une fonction de production augmentée, des modèles de correction d'erreurs de production ont été estimées. Les résultats indiquent que le taux de variation de la production de ciment est positivement lié aux exportations et négativement avec la concurrence étrangère. L'activité économique globale a montré un fort effet sur la production. Sous l'hypothèse que l'industrie fonctionne en oligopole, les résultats impliquent qu'il n'y a pas d'incitations pour favoriser l'entrée des concurrents.

Mots clés: industrie du ciment, la production, les entreprises, le marché, l'investissement, l'emploi.

\section{INDÚSTRIA DE CIMENTO NO MÉXICO: ANÁLISE DOS DETERMINANTES Resumo}

Abaixo se apresenta à análise e identificação de alguns determinantes que explicam a produçáa da indústria do cimento no México. O objetivo do estudo foi identificar o efeito das exportaçóes e da concorrência externa sobre a produção de cimento, assim como determinantes internos como o investimento, o emprego e a atividade econômica em geral. Com uma função de produção aumentada se estimaram modelos de correção de erros. Os resultados indicam a mudança da taxa de produção de cimento se relaciona positivamente com as exportaçóes e negativamente com a concorrência externa. A atividade econômica em geral mostrou um efeito forte sobre a produção. Supondo que a indústria opera em oligopólio, os resultados implicam que não existem incentivos para favorecer a entrada de concorrentes.

Palavras-chave: indústria de cimento, produção, negócios, mercado, investimento, emprego

墨西哥水泥行业的决定因素分析

贝伦・巴斯盖斯 萨尔瓦多・克拉莱斯

摘要

本文分析和阐述影响, 墨西哥水泥生产的决定因素。本文旨在探究出口和外 部竞争以及内部因素如投资、就业和整体经济活动等对水泥生产的影响。 本文借助误差修正模型进行分析研究。结果显示水泥生产变化率和出口成 正比, 和外部竞争成反比。整体经济活动对水泥生产有很大影响。本文在 水泥行业为垄断行业这一假设下进行分析, 结果显示在墨西哥没有有利于 竞争者进入的激励机制。

关键词：水泥行业, 产量, 企业, 市场, 投资, 就业 


\section{INTRODUCCIÓN}

La industria del cemento en México es una de las más importantes en cuanto a su capacidad productiva, sin embargo, a lo largo de los años ha tenido severas fluctuaciones en sus niveles de producción. El mayor impulso de esta industria ocurrió durante la época de industrialización por sustitución de importaciones que promovía la producción nacional a través de incentivos fiscales y apoyos financieros. La industria pasó de contar con una capacidad instalada de 574 millones a 17.02 millones de toneladas métricas en el periodo 1940-1980 (Salomón, 2006). Con seis grupos empresariales que integraban la industria, entre 1940 y 1960, la tasa de crecimiento anual fue de 10.2\%. El principal participante era Cementos Tolteca, seguido de Cementos Anáhuac, Cementos Mexicanos, Cruz Azul, Guadalajara y San Luis Mining Co. (Kumakan y Martínez, 2008).

A partir de la década de los noventa, las cementeras mexicanas realizaron importantes reacomodos mediante adquisiciones y fusiones que resultan de suma importancia para entender el reparto del mercado, el comportamiento de los precios y la competencia en los mercados internacionales en la actualidad. Información obtenida en el año 2015 mostraba que la industria del cemento todavía se encontraba altamente concentrada en un grupo de seis empresas que aún hoy dominan el mercado nacional: Cemex, Grupo Cementos de Chihuahua, Cemento Moctezuma, Holcim-Apasco, Cruz Azul y Cementos Fortaleza (constituida por la compra de Lafarge por Carlos Slim y Antonio del Valle, principal accionista del conglomerado químico Mexichem) (véase cuadro 1). Cemex domina el mercado nacional con cerca de $50 \%$ de la producción y ventas, seguida de Holcim-Apasco.

Existen pocos trabajos formales que hayan analizado a detalle el comportamiento de la industria cementera en México. Los más relevantes se han centrado en analizar las características generales de la industria (Avalos y Schatan, 2003; Kumakan y Martínez, 2008), en analizar la utilización eficiente de energía en la producción (Sterner ,1990), la estructura de mercado bajo la cual operan (De la Garza y Arteaga, 2012) y la relación de aglomeración y precios de las seis multinacionales (Ghemawat y Thomas, 2005). En otros trabajos se da énfasis al análisis solo de Cemex, dado su papel como empresa dominante y global (Barragán y Cerutti, 2003) y también al grupo integrado por Cemex, Holcim-Apasco y Lafarge (Vera, 2013).

De esta manera, el presente trabajo se concentra en analizar algunos de los determinantes de la producción y el crecimiento de la industria cementera en México. El objetivo es investigar en qué medida una mayor participación de 
Belem Vásquez y Salvador Corrales

Cuadro 1. Empresas que integran la industria del cemento en México (2015)

\begin{tabular}{lcl}
\hline Empresa & Número de plantas & \multicolumn{1}{c}{ Sede } \\
\hline Cemex & 15 & Monterrey, N. L. \\
Cemento Moctezuma & 3 & México, D.F. \\
Cementos y Concretos Nacionales (Cruz Azul) & 4 & México, D.F. \\
GCC Cemento & 3 & Chihuahua \\
Holcim-Apasco & 7 & México, D.F. \\
Cementos Fortaleza (Lafarge) & 3 & México, D.F. \\
\hline
\end{tabular}

Fuente: elaboración propia con datos de la Cámara Nacional del Cemento.

la industria en el mercado internacional, a raíz de sus planes de expansión y eliminación de tarifas antidumping han incrementado la producción y han podido medir el efecto de la competencia externa a través de las importaciones de cemento. Otro objetivo consiste en analizar los determinantes de índole interna como la inversión, el empleo y la actividad económica general sobre la producción. Partiendo de una función de producción aumentada se estimaron varios modelos de corrección de error para determinar la significancia estadística de cada uno de estos elementos de orden externo e internos sobre la producción de cemento en México, con datos mensuales a partir de 2005 hasta 2015.

El documento se compone de cinco apartados: el primero es un análisis teórico sobre la estructura de mercado del oligopolio, el segundo, un análisis de las tendencias en México con datos agregados de empleo, inversión y comercio exterior; un tercero se compone de la descripción de la metodología; un cuarto analiza los resultados obtenidos y finalmente el quinto apartado son conclusiones. Se asume como hipótesis que la creciente presencia de importaciones en el mercado nacional es un elemento que afecta la producción y que la eliminación de los aranceles no ha impulsado las exportaciones de cemento. A nivel del mercado interno, se asume que es el crecimiento de la industria de la construcción la principal determinante del aumento de la producción cementera. 


\section{DISCUSIÓN TEÓRICA}

La estructura de mercado, en la que opera la industria del cemento, se puede considerar como un oligopolio ya que impera un producto homogéneo y las empresas son interdependientes para fijar la producción o los precios. Las barreras impuestas a la entrada de nuevos competidores han hecho que existan bajos niveles de importación de cemento en México y, además, acusaciones sobre prácticas de comercio desleales han afectado también su capacidad de exportación a su principal mercado (Estados Unidos). En los últimos 10 años, la mayor tasa de crecimiento se alcanzó en 2006 con 7.7\%, mientras que el valor de la producción aumentó $5.8 \%$ en términos reales, sin embargo, es a partir de 2006 que la industria empieza a tener un fuerte declive en sus niveles de producción y de exportaciones. El momento más crítico ocurrió entre 2009 y 2010 cuando se registraron fuertes caídas en la producción de -4.21 y de $-52.4 \%$ en las exportaciones. Es comprensible que la crisis financiera desatada por Estados Unidos en el 2008 tuviera fuertes repercusiones en la economía mexicana y en particular en la industria cementera; sin embargo, mientras la economía en su conjunto se recuperaba en 2013, tanto la actividad de la construcción como la producción cementera siguieron presentando tasas negativas de crecimiento. Según el Instituto Nacional de Estadística y Geografía (INEGI), en 2015 la industria produjo 44.9 millones de toneladas de cemento y por primera vez en 10 años logró crecer a una tasa de 7\% (INEGI, 2015a).

A diferencia de la competencia perfecta, donde las empresas toman los precios como dados por el mercado y a partir de él establecen su nivel de producción, en el oligopolio la decisión que toma cada empresa afecta al precio de mercado y, por lo tanto, los beneficios de las demás empresas. Dado que producen un bien homogéneo, necesariamente existe interdependencia entre ellas y en consecuencia, su comportamiento debe ser estratégico.

En la teoría de la "competencia imperfecta" existen diversos modelos para explicar el comportamiento de los agentes en un oligopolio. Partiendo de un modelo simplificado donde existe $n$ número de empresas que producen un bien homogéneo, las empresas deben decidir el precio que cobra cada una o la cantidad que produce. Al maximizar los beneficios (ingreso menos costos), se pueden hallar dos soluciones: en el modelo de Cournot las empresas eligen simultáneamente la cantidad, mientras que en el modelo de Bertrand establecen simultáneamente el precio (Varian, 2011).

En el oligopolio el ingreso $\left(I T_{i}\right)$ y beneficio de la empresa $i\left(\Pi_{i}\right)$ están en función no sólo de su nivel de producción $\left(q_{i}\right)$, sino también, en función de la 
producción del resto de las empresas: $Q_{-i}$. De esta forma la función de beneficio para la empresa $i$ es:

$$
\Pi_{i}=I T_{i}\left(q_{i}, Q_{-i}\right)-C T_{i}\left(q_{i}\right)
$$

Donde $C T_{i}$ son los costos totales. Al considerar cuál debe ser su producción maximizadora $\left(q_{i}\right)$, la empresa $i$ debe considerar el comportamiento de las otras empresas $\left(Q_{-i}\right)$. A esta función se le denomina función de reacción pues representa su producción en términos de la producción de las otras empresas. Por lo tanto, en el equilibrio las cantidades de producción son iguales:

$$
q_{i}^{*}=F_{i}\left(q_{j}^{*}\right), q_{j}^{*}=F_{j}\left(q_{i}^{*}\right) \quad \text { donde } i, j=1, \ldots, n, i \neq j
$$

Por tanto, al maximizar, el precio del oligopolio es:

$$
P^{*}=(n c) /(n+1)
$$

El precio del producto depende del costo marginal $c$ y de $n$, por ello en el límite el precio es igual al costo marginal, es un precio competitivo:

$$
\lim _{n \rightarrow \infty} P^{*}(n)=c
$$

Si en el duopolio u oligopolio existen claras diferencias entre las empresas, y una de ellas domina, entonces existen dos posibles soluciones. Si la empresa dominante logra fijar el precio antes que la otra, se convierte en la líder en la elección de precio y, por lo tanto, la otra es la seguidora. Si lo que la empresa dominante fija es la cantidad, entonces es la líder en la elección de la producción y las empresas seguidoras maximizan sus beneficios a partir de esa producción (modelo de competencia Stackelberg).

Otras de las estrategias que las empresas pueden adoptar es la colusión, es decir, a través de la colaboración pueden reducir la competencia entre ellas para maximizar los beneficios como si fueran una sola empresa:

$$
{ }_{q_{1}, q_{2}}^{\operatorname{Max}} \pi_{1}+\pi_{2}=\left(q_{1}+q_{2}\right) P-C T_{1}\left(q_{1}\right)-C T_{2}\left(q_{2}\right)
$$


En la expresión (5), los beneficios totales son la suma de los beneficios de cada empresa. Los costos marginales para cada empresa son iguales al ingreso marginal de la industria. Dado que sólo se maximiza una función, el beneficio máximo de la industria es similar a lo que obtendría un monopolista con varias plantas. La ventaja de la colusión es que permite, mediante la fijación de la producción o el precio del producto, impedir la entrada de nuevos competidores. Sin embargo, el equilibrio en la colusión no es estable, pues las empresas requieren establecer un criterio para el reparto del beneficio total, por lo que si una de ellas no respeta los acuerdos, entonces puede aumentar su cuota de producción y los beneficios a costa de las demás. La colusión ocasiona que los beneficios totales de la industria sean mayores al fijar la producción o precios, pero ocurre en detrimento de los consumidores y de nuevos competidores. Por esta razón, la colusión es una práctica sancionada por las leyes de competencia de los gobiernos.

En la práctica es común que las empresas que acuerdan formar carteles, se mantengan por muchos años. Levenstein y Suslow (2006) hicieron una revisión extensa de casos empíricos de carteles en diferentes industrias para identificar, entre otras cosas, qué tan exitosos son y cuánto tiempo han durado. Encontraron que la duración es muy variada, mientras que el cartel de cerveza (en Estados Unidos) se mantuvo por nueve años consecutivos, el cartel del cemento (en Sudáfrica) logró mantenerse por 40 años (Levenstein y Suslow, 2006: 53) El éxito y duración de los carteles es mayor donde existe menor número de participantes y donde la demanda del producto es inelástica. En ambos casos, la industria cementera cumplió estas condiciones. Otros casos documentados parecen apoyar que la colusión y concentración de la industria del cemento son un tema común alrededor del mundo, por ejemplo, esto sucede en la India (Pradhan, 1992), Canadá (Kleit y Palsson, 1996) y Alemania (Blum, 2007), entre otros.

Además de las prácticas oligopólicas de competencia, existen otras teorías que explican el comportamiento de empresas para buscar reducir la competencia. Por ejemplo, la teoría de integración vertical sostiene que la adquisición de empresas que pertenecen a la cadena de producción y distribución conduce a una integración vertical, dando origen a la creación de barreras de entrada para los nuevos competidores (Blair y Kaserman, 1983; Carlton, 1979). Por ejemplo, Kleit y Palsson (1996) encontraron que, en el caso de las empresas cementeras del centro de Canadá, la adquisición de empresas integradoras y de distribución de cemento había creado una integración vertical que impedía la entrada de nuevos competidores pues implicaba altos costos para participar. La integración vertical de estas empresas permitía, por lo tanto, establecer precios elevados en comparación a empresas ubicadas fuera de la región. 


\section{DESEMPEÑO DE LA INDUSTRIA CEMENTERA MEXICANA}

La industria cementera mexicana oferta uno de los productos más importantes para la industria de la construcción: el cemento, cuya cadena productiva en la industria es muy corta (Pozas, 1999), ya que al hacerse las construcciones son muy pocas las modificaciones que se le pueden hacer para seguir agregando materiales complementarios. Desde otra perspectiva, aquí convergen varias cadenas (acero, vidrio, aluminio, madera, etcétera) hasta concluir las obras, cuyos usos definirán el tipo de materiales y, asimismo, las demandas de cemento.

Por la composición de los materiales e insumos que necesita esta industria, se ha concentrado en unas pocas empresas hasta crear un mercado oligopólico, propiciando prácticas de colusión abiertas y encubiertas para manipular el mercado. Dada la estrechez de su cadena productiva y especialización en unos cuantos productos, las empresas no invierten en actividades industriales complementarias. La experiencia de Cemex ha sido un ejemplo de éxito que desafía la lógica de las redes de empresas transnacionales, que formatean la globalización de la economía (Corrales, 2006; Kang y Sakai, 2000).

Es más común que los industriales del cemento realicen fusiones y adquisiciones para controlar el mercado, lo que propicia la competencia internacional mediante compras hostiles de empresas y de cementeras pequeñas que están bien posicionadas en mercados en crecimiento y con demanda. En las últimas dos décadas, la estrategia de aglomeración (Ghemawat y Thomas, 2005), ha hecho patente este proceso de concentración en la industria cementera, cuyos beneficiaros finales han sido las grandes empresas transnacionales del cemento.

En México y en el mundo, las grandes obras de infraestructura como puentes y edificios demandan importantes cantidades de cemento para realizarse y propician la competencia entre las empresas cementeras para abastecerlas. Seguramente, por la alta composición del capital para producirlo y el costo de transporte para llevarlo hasta el consumidor final, la industria cementera se encuentra dominada por sólo algunas empresas, es decir, tiene capacidad de manipular los precios (De la Garza y Arteaga, 2012).

En el cuadro 2 puede apreciarse algunos indicadores del desempeño de la industria cementera ${ }^{2}$ desde 2005 hasta 2015. A lo largo de estos años, la industria ha estado controlada por seis empresas que poseen alrededor de 34 a 37 plantas. Actualmente cuentan con 30 mil puntos de venta a lo largo de

Los datos de la industria cementera se refieren a los datos clasificados por INEGI en la rama 327310 :

"Fabricación de cemento y productos a base de cemento en plantas integradas". 
todo el territorio nacional, generando 86 mil empleos directos; sin embargo, su participación en el Producto Interno Bruto (PIB) fluctúa alrededor de 1\%. En el cuadro observamos que en 2005 la producción de cemento nacional fue de 39.7 millones de toneladas, mientras que el consumo nacional per cápita fue de 384.1 kilos por habitante: 10 años más tarde, aunque la producción fue mayor (44.9 millones de toneladas) el consumo per cápita se redujo a 375.5 kilos por habitante.

Los efectos de la crisis financiera de 2008 muestran cómo el consumo per cápita durante el 2009 alcanzó 396 kilogramos, que bien puede ser explicado por las presiones de la recuperación a través de nuevas inversiones en infraestructura e ingeniería, que tienen comportamientos semejantes a la tasa de crecimiento de la economía nacional. En vista del poco crecimiento económico después de la crisis, muy dependiente de la economía norteamericana, la caída del consumo nacional y per cápita de cemento se hace evidente. Una

Cuadro 2. Producción y consumo de cemento en México

\begin{tabular}{lcccccc}
\hline Año & $\begin{array}{c}\text { Número de } \\
\text { plantas } \\
\text { a) }\end{array}$ & $\begin{array}{c}\text { Capacidad } \\
\text { utilizada } \\
\text { promedio } \\
(\%)\end{array}$ & $\begin{array}{c}\text { Producción } \\
\text { cemento } \\
\text { (millones } \\
\text { de ton.) }\end{array}$ & $\begin{array}{c}\text { Tasa de } \\
\text { crecimiento, } \\
\text { producción } \\
(\%)\end{array}$ & $\begin{array}{c}\text { Consumo } \\
\text { per cápita } \\
\text { (kilos por } \\
\text { hab.) }\end{array}$ & $\begin{array}{c}\text { Valor de la producción } \\
\text { (mill. de pesos } \\
\text { de 2008) }\end{array}$ \\
\hline 2005 & 37 & 84.1 & 39.7 & & 384.1 & 59828.2 \\
2006 & 37 & 86.3 & 42.7 & 7.7 & 413.7 & 63316.9 \\
2007 & 35 & 85.7 & 43.4 & 1.6 & 420.5 & 62352.0 \\
2008 & 35 & 85.8 & 42.3 & -2.6 & 409.5 & 59816.2 \\
2009 & 35 & 85.0 & 40.9 & -3.2 & 396.3 & 58186.5 \\
2010 & 35 & 83.3 & 39.2 & -4.2 & 349.2 & 56034.8 \\
2011 & 35 & 84.2 & 40.6 & 3.6 & 361.6 & 55201.2 \\
2012 & 34 & 83.9 & 41.6 & 2.5 & 370.5 & 56229.2 \\
2013 & 35 & 80.6 & 39.8 & -4.3 & 354.6 & 57189.8 \\
2014 & 34 & 82.2 & 41.9 & 5.3 & 373.3 & 60026.8 \\
2015 & 34 & 84.3 & 44.9 & 7.0 & 375.5 & 64047.4 \\
\hline
\end{tabular}

a/ El número de plantas de 2005 y 2007 proviene de la EalM, Scian 2002.

Fuente: elaboración propia con datos de la EMIM, datos anuales y Censo de Población, INEGI. 
revisión a mayor detalle, con estadísticas de la Encuesta Mensual de la Industria Manufacturera (EMIM) del INEGi (2015b), muestran la caída en el valor de la producción desde el 2006, año que significó el nivel máximo previo a la crisis financiera global, al registrar una tasa de crecimiento real de $5.8 \%$. De 2008 a 2010 sólo se registraron tasas de crecimiento negativas. Es posible que el efecto de la crisis financiera haya pegado fuertemente a los productores de cemento, ya que Cemex, Holcim-Apasco y Lafarge mantienen altos montos de colocación en los mercados bursátiles para financiar su actividad productiva (Vera, 2013).

La situación en 2015 fue diferente, ya que la industria mostró entonces rápidos signos de recuperación. Ese año el valor de la producción ascendió a 64 mil millones de pesos (a precios de 2008), lo que representó un crecimiento real de 6.7\%. Los datos de la EMIM confirman que en 2015, la capacidad productiva del cemento fue de 45 millones de toneladas y tuvo una capacidad ociosa de $15.7 \%$. En cuanto a las características del producto, la mayor parte de la producción de cemento es el portland que se elabora con material clinker pulverizado (representa más de $80 \%$ de la producción total). En orden de importancia le sigue el cemento blanco, que es la mezcla de piedra caliza, arcilla de caolín con bajo contenido de hierro y yeso (representa alrededor de $2.5 \%$ ) y el mortero que es la mezcla de cemento con arena y agua (alrededor de $8 \%$ ). Estos tres tipos de materiales constituyen los principales productos de la industria. También existe el cemento mixto que es la integración de cemento portland y escoria molida y granulada de los altos hornos, cenizas volátiles, humos de silicato, arcilla calcinada, caliza hidrogenada y otras puzolanas (Cemex, 2016). En el cuadro 3 se presenta cómo ha fluctuado la participación de cada tipo de cemento a lo largo del periodo.

Con respecto a la creación de empleos, ha fluctuado de manera cercana con la producción; sólo que por ser una industria intensiva en capital, el empleo de años recientes es mucho menor al que se registró en 2005. La industria ha realizado importantes inversiones a lo largo del periodo, a excepción de la crisis de 2009-2010. Las expectativas de comercio internacional, así como la presencia de nuevos productores (Cementos Fortaleza, de Carlos Slim y asociados) dieron un impulso a la inversión en los últimos ańos: de $116.2 \%$ en 2015, podría ser resultado también de un incremento del consumo, que según la Cámara Nacional del Cemento (CANACEM) fue de 7.6\%; mientras que los datos del INEGI reportaron un $9.6 \%$.

Como puede observarse en la gráfica 1, están representadas las tasas de crecimiento anual de algunos indicadores ya mencionados. Se percibe la tendencia generada por el ciclo de recuperación en la industria cuyo costo ha sido 
Cuadro 3. Principales indicadores de la industria del cemento en México. Periodo 2005-2015

\begin{tabular}{lcccccc}
\hline Año & $\begin{array}{c}\text { Producción total } \\
\text { (millones } \\
\text { de tons.) }\end{array}$ & Portland (\%) & Blanco (\%) & Mortero (\%) & $\begin{array}{c}\text { Personal } \\
\text { ocupado }\end{array}$ & $\begin{array}{c}\text { Inversión fija bruta } \\
\text { (millones de } \\
\text { pesos) }\end{array}$ \\
\hline 2005 & 39.7 & 90.7 & 2.0 & 7.4 & 92943 & 1394.6 \\
2006 & 42.7 & 84.2 & 2.0 & 7.4 & 93668 & 3026.0 \\
2007 & 43.4 & 82.8 & 2.0 & 7.8 & 93960 & 2348.4 \\
2008 & 42.3 & 85.1 & 1.9 & 8.2 & 92237 & 3206.7 \\
2009 & 40.9 & 87.9 & 1.6 & 8.4 & 84708 & 1202.6 \\
2010 & 39.2 & 91.8 & 1.8 & 6.7 & 84105 & 1816.4 \\
2011 & 40.6 & 88.6 & 1.9 & 8.7 & 87471 & 1065.2 \\
2012 & 41.6 & 86.5 & 2.1 & 9.1 & 87967 & 4478.6 \\
2013 & 39.8 & 90.4 & 2.4 & 9.0 & 87382 & 1852.8 \\
2014 & 41.9 & 85.8 & 2.7 & 9.0 & 86022 & 4365.3 \\
2015 & 44.9 & 80.2 & 2.0 & 9.3 & 86802 & 4278.0 \\
\hline
\end{tabular}

Fuente: elaboración propia con datos de la EMIM y EAIM del INEGI y Secretaría de Economía.

Gráfica 1. Tasas anuales de crecimiento (\%)

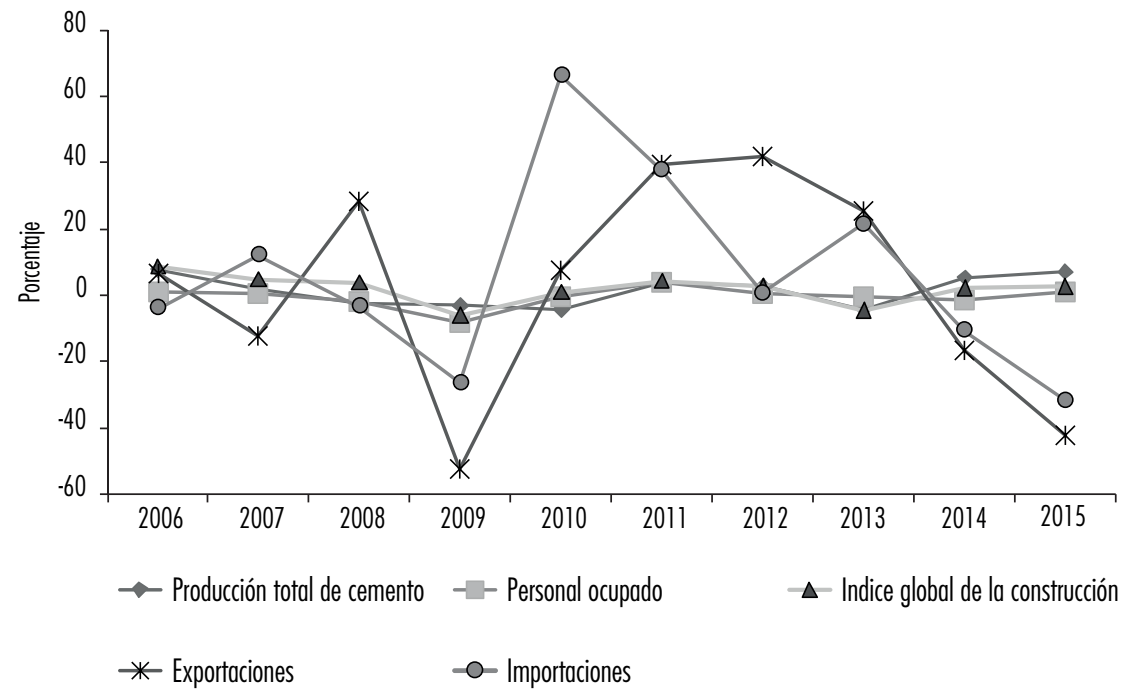

Fuente: elaboración propia con datos de la EMIM (INEGI, 2015b), INEGI (2015a) y Secretaría de Economía (2016). 
la caída en la tasa de crecimiento del personal ocupado, la producción y el comercio internacional en 2009. Así como la cercana relación entre el indicador de la actividad económica de la construcción con el cemento, pues la construcción representa la principal demandante de cemento y concreto premezclado.

En julio de 2014, la fusión de Lafarge y Holcim-Apasco, puso en jaque a todos los competidores inclusive de México, por la recomposición de la industria con nuevos y viejos competidores para repartirse el mercado, con prácticas oligopólicas que afectan el precio. La presencia en esta industria de uno de los empresarios más ricos de México y del mundo, la situación de estancamiento en la industria de la construcción mexicana, junto con crecientes importaciones tanto de Estados Unidos como de China, han hecho bajar los precios del cemento en algunas regiones del país (Torres, 2014).

Las exportaciones de cemento mexicano por la composición de su valor, registraron un repunte importante a partir de 1985, con ingresos superiores a los 150 millones de dólares, pero en 1990 cayeron por abajo de los 70 millones, en respuesta a las leyes antidumping aplicadas por Estados Unidos en 1989. La firma del Tratado de Libre Comercio con Norteamérica (TLCAN) en 1994 representó un gran avance en las negociaciones comerciales; sin embargo, la industria cementera mexicana no tuvo beneficios pues al mismo tiempo recibía sanciones antidumping. Estas sanciones eran cuotas de hasta 26.28 dólares por tonelada de cemento, lo que hizo difícil exportar a precios competitivos.

Para los analistas estadounidenses el precio del cemento es ampliamente determinado por los costos del transporte; no obstante, los precios del cemento mexicano se ubicaban por debajo de los precios americanos y había que transportarse a grandes distancias, lo que se tenía que traducir a precios más altos (Cook, 1994). Esto se reflejó en impuestos compensatorios de entre 43 y $62 \%$ en plena crisis de 1995 . Cemex emprendió una nueva estrategia comercial: consolidarse en la nación norteamericana como una unidad de negocios autónomo, para no depender de las exportaciones (Barragán y Cerutti, 2003). Generalmente las exportaciones como proporción de la producción total no son elevadas y las estrategias de competencia se basan en las fusiones y adquisiciones o colusiones. Como resultado de la competencia desleal de Cemex y de otras compañías del mundo, de existir 141 plantas operando en Estados Unidos durante 1986, su número se redujo a 134 en 1989 y 67 de dichas plantas fueron propiedad de extranjeros o eran operadas en Joint ventures (Cook, 1994). Por el contrario, en 2006 Cemex ya tenía 12 plantas en Estados Unidos con capacidad de 13.2 millones de toneladas (Salomón, 2006). Algunos estudios prueban que las medidas antidumping aplicadas al cemento 
portland generaron efectos diferenciados en Estados Unidos debido a la segmentación del mercado. Cohen-Meidan (2013) encontró que en la región de Norteamérica cercana al Golfo de México, las medidas antidumping aplicadas al cemento mexicano aumentaron los precios domésticos $9 \%$ más que el grupo de control; sin embargo, no alteraron los precios en la región fronteriza ni en California. Esto sugiere, por lo tanto, que las exportaciones mexicanas perdieron mercado principalmente en las regiones donde no tenían competitividad sus exportaciones, mientras que mantuvieron el dominio en las que proveían a través del transporte marítimo (cuyo costo es menor al terrestre).

Después de 16 años de disputas entre México y Estados Unidos, en 2006 se lograron acuerdos para establecer diferentes periodos para permitir la exportación de cemento mexicano a regiones específicas. En estas negociaciones se acordó que se podría exportar cemento gris portland y cemento clinker no mayor de 3 millones de toneladas, iniciando el 3 de abril de 2006 y concluyendo el 31 de marzo de 2007. El segundo periodo se fijó del 1 de abril de 2007 al 31 de marzo de 2008 y el tercero del 1 de abril de 2008 al 31 de marzo de 2009 (Secretaría de Economía, 2006). Una vez vencido el acuerdo a principios de 2009, y sin ninguna controversia presentada ante el Departamento de Comercio de Estados Unidos, se eliminaron las cuotas, así como los límites de exportación (US Department of Commerce, 2009).

De acuerdo a datos del Sistema de Información Arancelaria Vía Internet (siavi) de la Secretaría de Economía, en 2005 las exportaciones de cemento portland se enviaron a Estados Unidos (87.86\%), a Guatemala (4.1\%), a Belice (4.3\%), a Panamá (2.9\%) y al resto del mundo (0.84\%). Con respecto a las importaciones, tanto en valor como en volumen para ese mismo año, casi $61 \%$ provinieron de Estados Unidos, es decir, prevalece el comercio intra-industrial. Dada la relación peso-precio del cemento, el monto de las exportaciones es relativamente pequeño con respecto a la producción nacional (cercano a 3\%).

Las importaciones de cemento tienen mucho menor peso en la estructura del comercio; por su origen, hasta el 2015 han mantenido la misma tendencia de socios comerciales, $77.1 \%$ de las importaciones de cemento llegaron de Estados Unidos, $15.1 \%$ de China y 3.6\% de Croacia (Secretaría de Economía, 2016). Al mercado nacional también ingresan importaciones de otros países como: Espańa, Países Bajos, Polonia y Alemania, entre las más frecuentes. La controversia comercial con Estados Unidos por las medidas antidumping tuvo un efecto importante en sus exportaciones, no obstante, por la capacidad productiva de la industria cementera mexicana, el saldo de la balanza comercial se mantuvo positivo (véase gráfica 2). 
Gráfica 2. Comercio internacional de la industria cementera y participación de la competencia en el mercado nacional

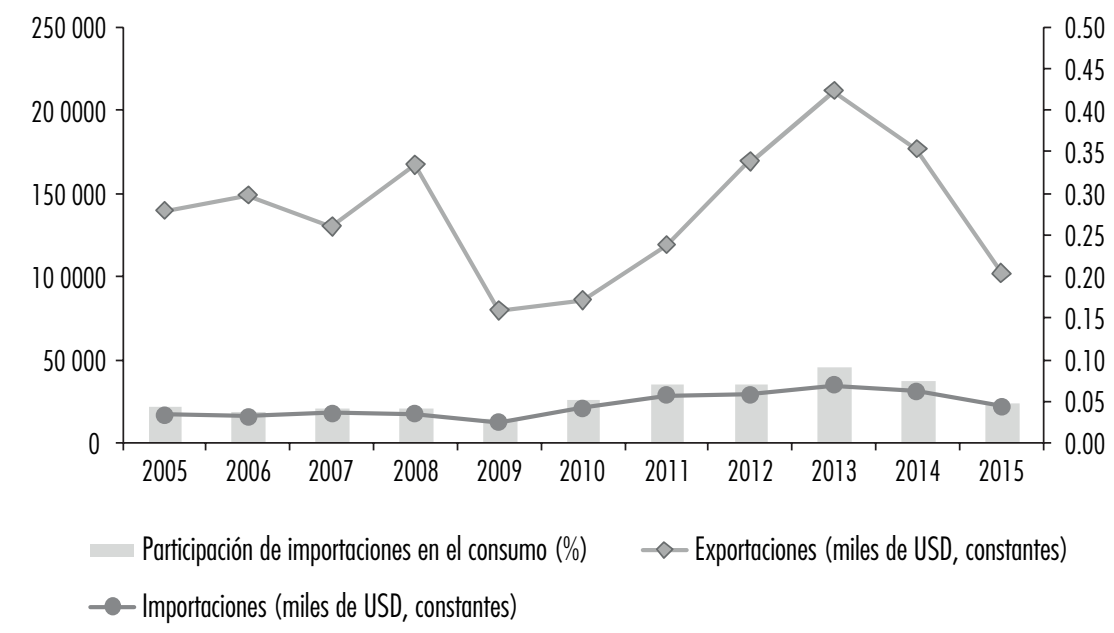

Fuente: elaboración propia con datos del INEGI (2015b) y Secretaría de Economía (2016).

En la gráfica anterior se presentan cifras -en el eje derecho- que representan la participación de la competencia extranjera medida a través del porcentaje de importaciones con respecto al consumo nacional de cemento. A partir de 2009, la participación de la competencia externa ha ido en aumento, llegando a su máximo nivel en 2013. Dado que la industria nacional tiende a ser poco abierta a la competencia, es importante resaltar que aunque aparentemente la industria tiene capacidad para abastecer la demanda, existen inconformidades del consumidor que propicia la demanda de cemento importado.

En virtud de lo anterior, podemos recordar el caso del barco Mary Nour que intentó descargar 27 mil toneladas de cemento ruso a mediados de 2004. Los protagonistas de este desafío corresponde a un grupo de emprendedores mexicanos que confiaron en la apertura comercial. Para ese propósito crearon la empresa Comercio para el Desarrollo Mexicano, cuyo objetivo era importar 500 toneladas anuales de cemento por el Golfo de México y una cifra similar por el Océano Pacífico (Torres, 2004; García, 2004). El argumento de estos nuevos comerciantes de cemento consistió en que los precios del cemento mexicano son de los más caros del mundo (De la Rosa, 2016); sin embargo, las autoridades del puerto de Tampico, Tamaulipas, les negaron el desembarco en atención a un amparo emitido por Cemex, según el cual la embarcación 
podría dañar el canal de navegación del puerto. En resumen, la importación no pudo realizarse.

Este hecho se tradujo en una victoria para la industria cementera nacional dirigida por Cemex, que establece precios por encima de los precios internacionales, permitiéndoles aumentar sus beneficios y así consolidarse por el mundo entero (Salomón, 2006; Ghemawat y Thomas, 2005; García, 2004). Los ejecutivos de Cemex justifican sus precios por las características del mercado mexicano: un 75\% del cemento se vende en sacos de 50 y 25 kilos y no a granel, lo cual exige una mayor logística de distribución y costos de transporte que elevan el precio. Otras fuentes de información sostienen que estos privilegios oligopólicos de las empresas cementeras mexicanas, propician utilidades cercanas a 300\%. "La utilidad porcentual de la comercialización del cemento para el mercado interno es de $271 \%$, mientras que para el mercado externo se ubica entre $152 \%$ y $155 \%$ cifra que permite observar una variación porcentual por encima del $100 \%$ en el mercado nacional, lo cual favorece al mercado externo" (Hernández Pérez, 2004: 3).

En un análisis de las ganancias obtenidas por la aglomeración de seis grandes empresas multinacionales (Lafarge, Holcim-Apasco, Cemex, Heidelberger, Italcementi y Blue Circle Industries), Ghemawat y Thomas (2006) encontraron que la participación de las multinacionales en la capacidad total en un país genera aumento del precio promedio por tonelada y la rentabilidad operativa. El aumento de rentabilidad de las cementeras no es por reducción de costos o por aumentos de eficiencia, sino por los incrementos de precios que se establecen cuando entran al mercado. Según estos autores, para el caso de México, la entrada de las multinacionales Holcim-Apasco y Lafarge en 1989, la expansión de Cemex de empresa local a global, propiciaron incrementos reales en los precios del cemento mexicano desde entonces. Aunado a ello, la mayor apertura comercial de México no ha propiciado mayor competencia y reducción del monopolio en la industria cementera. Shantayanan y Rodrik (1989) mostraron con un modelo de simulación, que el impacto de la liberalización comercial puede ser positivo en presencia de competencia imperfecta, sin embargo, esto tiende a ocurrir en la industria manufacturera y no en industrias con menor valor agregado.

\section{PROCEDIMIENTO ECONOMÉTRICO}

Aunque los agentes participantes de la industria del cemento siguen un comportamiento de competencia oligopólica, a nivel agregado la industria puede ser representada por una sola función de producción $Q$. De esta forma, para 
alcanzar el objetivo de determinar el efecto de los factores de orden externo (exportaciones y competencia de las importaciones) y el efecto de los internos (trabajo inversión y demanda) sobre la producción de cemento, se especificó la siguiente función de producción aumentada:

$$
\begin{aligned}
& Q_{t}=[L, K, \mu] \\
& Q_{t}=A_{t} L^{\alpha} K^{\beta} \mu^{1-\alpha-\beta}
\end{aligned}
$$

$Q_{t}$ es el valor de la producción de cemento total, $K$ es la inversión fija bruta, $L$ es el número de personal ocupado en la industria, $A$ es la eficiencia productiva y $\mu$ es la externalidad generada por la demanda internacional de cemento (para esto usamos las exportaciones como un proxy). Los coeficientes $\alpha$ y $\beta$ son la participación de capital y trabajo respectivamente y $(1-\alpha-\beta)$ es la participación de la demanda exportadora. Se asume que $\alpha$ y $\beta$ son menor a uno, de tal manera que hay retornos decrecientes de trabajo y capital.

Aplicando logaritmos naturales a la ecuación 7 y derivando con respecto a $t$, obtenemos la siguiente función de producción:

$$
\psi_{y}=\psi_{A}+\alpha \psi_{L}+\beta \psi_{K}+(1-\alpha-\beta) \Psi_{\mu}
$$

donde $\psi$ es la tasa de crecimiento de $i=Q, L, K, \mu$. La ecuación 8 implica que incrementos en la demanda de exportaciones aumentará la producción en $(1-\alpha-\beta)$. La ecuación puede tomar la siguiente forma:

$$
q=d_{0}+d_{1} l+d_{2} \text { inv }+d_{3} \text { ex }+d_{4} \text { compes }+d_{5} \text { igaet }+d_{6} D+\varepsilon
$$

En la ecuación 9, las letras en minúsculas indican logaritmos naturales, $\Delta$ es el operador de diferencias, $q$ es el valor de la producción de cemento, $l$ es el empleo, inv es la inversión fija bruta, ex es el valor de las exportaciones, compes es un indicador de la participación de la competencia externa en el mercado nacional y se mide como la participación de cemento importado en el mercado, igaet representa la actividad económica y se mide por un indicador global de actividad económica, $D$ representa una variable dummy que captura los efectos antidumping (toma el valor de 0 para 2005 a mayo de 2009 y de 1 a partir de abril de 2009), y $\mathcal{E}$ es el término de error. 
La base de datos proviene de la Encuesta Mensual de la Industria Manufacturera (ЕмIM) y de la Encuesta Industrial Mensual Ampliada (EIMA), publicadas por el INEGI (2015b, 2015c). El periodo de análisis considerado fue de 2005:1 a 2015:12. Los datos en pesos fueron deflactados a fin de eliminar el efecto de los precios, para ello se utilizó el Índice Nacional de Precios al Productor de Cemento, con base en junio de 2012. Posteriormente se convirtieron a dólares. Los datos originales en dólares fueron deflactados con el Índice de Tipo de Cambio Real, publicados por el Banco de México. Finalmente, todas las variables se transformaron a logaritmos naturales para reducir la varianza, esto se indica con una "l" agregada en la notación.

\section{RESULTADOS}

La utilización de series de tiempo mensuales, relativamente largas, requiere la aplicación de pruebas de raíz unitaria a fin de evitar realizar estimaciones de relaciones espurias que puedan conducir a inferencias inválidas. Por ello, se aplicaron pruebas de raíz unitaria a todas las variables, en específico las de Dickey Fuller Aumentada (1981) y Phillips y Perron (1988). Los resultados se presentan en el cuadro 4.

Ambas pruebas indican que la mayoría de las variables no son estacionarias en niveles, pero sí lo son en primeras diferencias. Por lo tanto, se puede considerar que están integradas de orden uno, I(1). Dado que las variables son I(1) es necesario aplicar la técnica de Johansen-Joselius (1990) para detectar si existe cointegración entre ellas. Si este es el caso, entonces en lugar de estimar la ecuación tal y como aparece en (9), es necesario agregar un mecanismo de corrección de errores. Para la aplicación de la prueba de Johansen, se seleccionó un vector autoregresivo (VAR) al que se le aplicaron pruebas a los residuos para detectar problemas de correlacion serial, heteroscedasticidad, normalidad y estabilidad matemática. El número de rezagos óptimo fue de cuatro, por lo tanto la prueba de cointegración se realizó con tres rezagos. En el el cuadro 5 se presentan los resultados.

Los estadísticos de máximo eigenvalor y de traza indican el rechazo de la hipótesis nula de no cointegración, lo que significa que se acepta la hipótesis alternativa de que existe al menos un vector. Una vez comprobada la existencia de cointegración, se procede según el teorema de Engle Granger (1987) a estimar un modelo de corrección de errores (MCE). Este modelo requiere introducir los residuos estimados de la ecuación de cointegración (de largo plazo) con un rezago, en la ecuación de corto plazo. El MCE sólo se estima 
Belem Vásquez y Salvador Corrales

Cuadro 4. Resultados de las pruebas de ráiz unitaria ADF y PP

Dickey Fuller Aumentada

Phillips-Perron

\begin{tabular}{|c|c|c|c|c|c|c|}
\hline Variables & & Level & Diferencia & & Level & Diferencia \\
\hline lq & \multirow{7}{*}{$\begin{array}{l}\text { Constante y } \\
\text { tendencia }\end{array}$} & 0.6883 & $-8.094^{\star}$ & \multirow{7}{*}{$\begin{array}{l}\text { Constante y } \\
\text { tendencia }\end{array}$} & $-6.125^{\star}$ & $-34.783^{\star}$ \\
\hline$\|$ & & -2.98 & $-10.8783^{\star}$ & & -2.901 & $-14.302^{\star}$ \\
\hline linv & & -2.8723 & $-7.4134^{\star}$ & & $-5.179^{\star}$ & $-10.038^{*}$ \\
\hline lex & & -2.9346 & $-12.0427^{\star}$ & & $-5.215^{*}$ & $-21.72^{*}$ \\
\hline Icompes & & -1.909 & -12.9111 * & & $-5.925^{\star}$ & $-27.942^{\star}$ \\
\hline ligat & & -1.9802 & -2.2819 & & $-5.865^{\star}$ & $-27.987^{\star}$ \\
\hline ligac & & -3.4093 & -2.7902 & & $-4.805^{\star}$ & $-19.928^{\star}$ \\
\hline Ivpro & \multirow{7}{*}{$\begin{array}{l}\text { Solo } \\
\text { constante }\end{array}$} & -0.5247 & $-3.5660^{\star}$ & \multirow{7}{*}{$\begin{array}{l}\text { Solo } \\
\text { constante }\end{array}$} & $-6.389^{\star}$ & $-34.644^{\star}$ \\
\hline IL & & -2.416 & $-10.906^{*}$ & & -2.135 & $-14.300^{*}$ \\
\hline linv & & -3.0008 & $-7.468^{\star}$ & & $-5.369^{\star}$ & $-10.128^{\star}$ \\
\hline lex & & -3.0068 & $-12.063^{*}$ & & $-5.258^{\star}$ & $-21.705^{\star}$ \\
\hline Icompes & & -1.8973 & $-12.922^{\star}$ & & $-4.759^{\star}$ & $-27.244^{\star}$ \\
\hline ligaet & & -0.2606 & -2.293 & & -1.959 & $-28.154^{\star}$ \\
\hline ligaec & & $-3.255^{\star \star}$ & -2.677 & & $-3.769^{*}$ & $-19.746^{*}$ \\
\hline
\end{tabular}

* Nivel de significancia de 1\%. El valor crítico es -4.03; *夫 Nivel de significancia de 5\%. El valor crítico es de -3.44.

Fuente: elaboración propia.

Cuadro 5. Resultados de la prueba de cointegración de Johansen. Intervalo: 1 a 3 rezagos

\begin{tabular}{cccccc}
\hline $\begin{array}{c}\text { Hipótesis } \\
\text { nula }\end{array}$ & $\begin{array}{c}\text { Hipótesis } \\
\text { alternativa }\end{array}$ & $\begin{array}{c}\text { Máximo } \\
\text { eigenvalor }\end{array}$ & $\begin{array}{c}\text { Valor crítico } \\
95 \%\end{array}$ & Traza & \multicolumn{1}{c}{$\begin{array}{c}\text { Valor crítico } \\
95 \%\end{array}$} \\
\hline 0 & 1 & $52.040^{\star \star}$ & 46.231 & $129.921^{\star \star}$ & 125.615 \\
$\leq 1$ & 2 & 29.016 & 40.078 & 77.881 & 95.754 \\
$\leq 2$ & 3 & 19.210 & 33.877 & 48.865 & 69.819 \\
$\leq 3$ & 4 & 15.660 & 27.584 & 29.656 & 47.856 \\
\hline
\end{tabular}

**Nivel de significancia de $5 \%$.

Fuente: elaboración propia. 
con variables estacionarias, así que se diferenciaron todas las variables dependientes como independientes, por lo que ahora representan tasas de cambio. En el cuadro 6 se presentan los resultados de las estimaciones de la ecuación: $q=d_{0}+d_{1} l+d_{2}$ inv $+d_{3}$ ex $+d_{4}$ compes $+e c m_{t-1}+\varepsilon$.

Los resultados de la estimación del modelo 9.1 indican que la tasa de cambio de la producción de cemento $(\mathrm{dq})$ está positivamente relacionada con el cambio en las exportaciones (dex). Es decir, el estímulo a las exportaciones de cemento ha sido un elemento fundamental que ha afectado positivamente el crecimiento de la producción de la industria en este periodo. Aunque una proporción menor de la producción cementera se destina a las exportaciones, esto parece ser un determinante importante de crecimiento, pues quizás implica mayor inversión en los procesos, mayor eficiencia en el uso de recursos, etcétera, de tal manera que aumentan el valor de la producción. También encontramos que la tasa de cambio en la competencia (dcompes), medida por la participación de las importaciones, es un elemento que impacta negativamente a la tasa de producción cementera nacional. Esto indica que, dada la estructura de mercado de la industria, las empresas que integran el oligopolio tienen pocos o nulos incentivos para permitir la mayor participación de competidores en el mercado. Este resultado sugiere que es posible que la industria mexicana favorezca las barreras a la entrada de nuevos competidores, lo cual estaría en correspondencia con los trabajos que han identificado la existencia de carteles en la industria cementera en otros países con el objeto mantener el poder de mercado (Pradhan 1992; Kleit y Palsson, 1996; Blum, 2007; Levenstein y Suslow, 2006). Con el marco de referencia de la teoría del oligopolio, De la Garza y Arteaga (2011) trataron de identificar la naturaleza del comportamiento de la industria cementera en México. Con datos de 2005 a 2007, los autores no hallaron evidencia de colusión, sino de competencia tipo Stalkelberg, pero estas estimaciones no fueron robustas. En cuanto al efecto de las variables de orden nacional, la tasa de crecimiento del empleo y de inversión fija no resultaron estadísticamente significativas.

En la estimación del modelo 9.2 se agregó una variable ficticia (dummy) para medir el impacto que tuvo la eliminación de las barreras arancelarias impuestas por Estados Unidos, en particular a partir de abril de 2009 fecha en que quedaron totalmente libres. En este caso, el coeficiente de la dummy resultó positivo más no significativo, lo que indica que la apertura al mercado estadounidense, aunque con signo correcto, no afectó la tasa de crecimiento del valor de la producción. El desempeńo de la industria parece haber estado ajena las sanciones impuestas por Estados Unidos, quizá por las estrategias empresariales que consideran al mercado interno como el principal destino de 


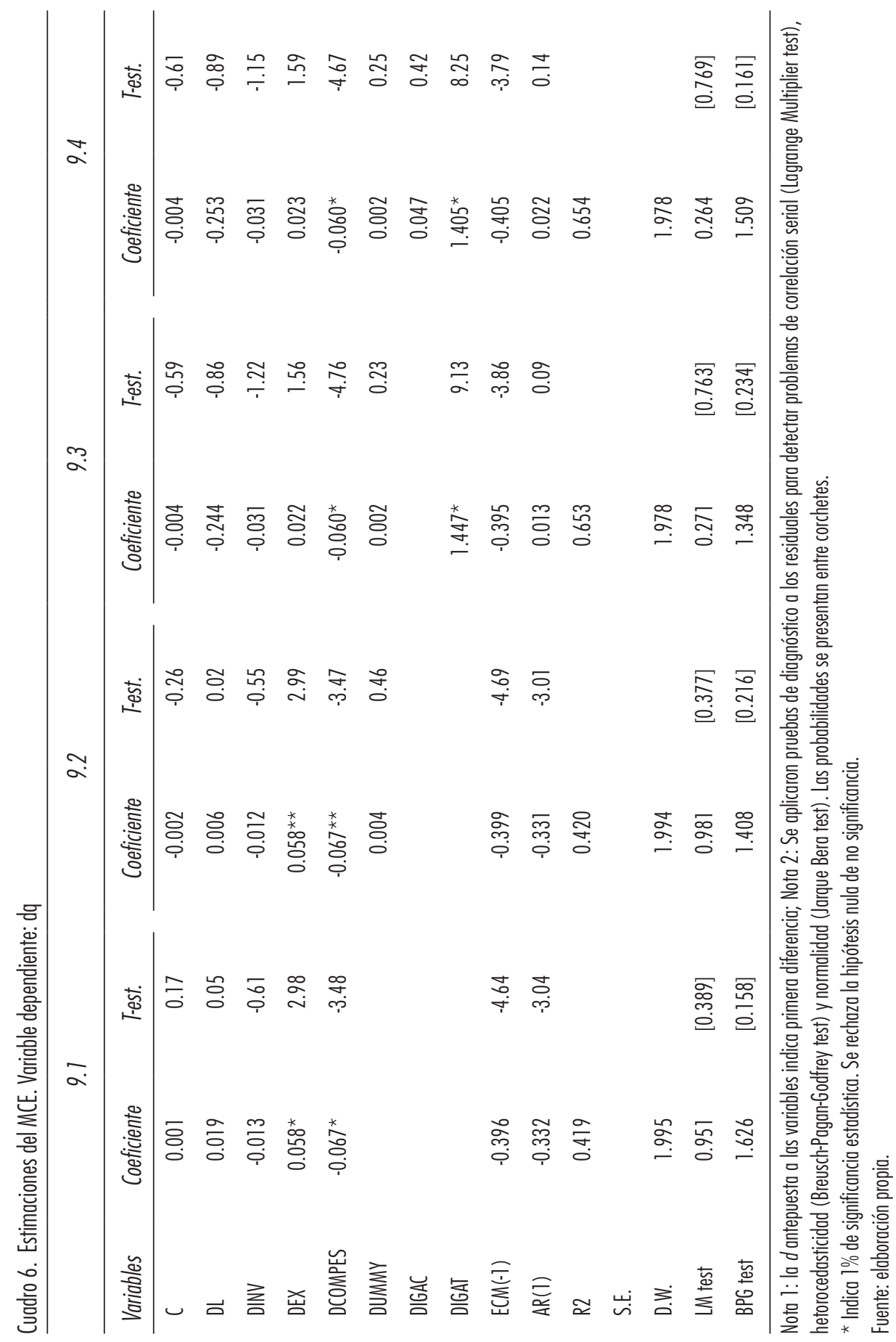


su producción, pero también porque precisamente cuando se eliminaron las barreras arancelarias en 2009, Estados Unidos entró en fuerte recesión por la crisis financiera.

En las estimaciones de los modelos 9.3 y 9.4, se incorporaron dos variables de orden nacional para determinar si la actividad económica en general y la de la industria de la construcción son explicativas del crecimiento del valor de la producción. De esta forma se añade la variable igat que es un indicador global de la actividad económica total e igac, que es un indicador global de la actividad económica, pero solo de la industria de la construcción. Ambas variables se miden a través de un índice de volumen físico de la producción calculado por el INEGI y que permiten medir los cambios en la producción en el corto plazo. La estimación del modelo 9.3 muestra que la tasa de cambio de la variable digat tiene un efecto positivo y significativo sobre la tasa de crecimiento de la producción de cemento igual a 1.447. Esto indica que el crecimiento de la actividad económica en general genera mayor demanda de cemento y, por ello, se identifica el efecto sobre la producción de cemento, pues incluye no sólo la construcción comercial e industrial sino también la autoconstrucción, ampliación y mejoramiento de infraestructura y demás actividades que utilizan al cemento como materia prima.

En el modelo 9.4 se añadió la variable digac para determinar si, en específico, es el crecimiento de la industria de la construcción la que impacta la tasa de cambio de la producción cementera. Los resultados indican que nuevamente es la actividad económica total digat, y no sólo la construcción, la que tiene un efecto estadísticamente significativo sobre el valor de la producción cementera. Gran parte de la demanda de cemento es para la creación de infraestructura en proyectos del gobierno y representa de 30 a $40 \%$ de la demanda, alrededor de $40 \%$ es para autoconstrucción de vivienda y desarrolladoras y alrededor de $20 \%$ es para construcción comercial e industrial. Por ello, es evidente que la producción y venta de cemento dependa de las condiciones de la economía, mayor gasto del gobierno federal, mayores créditos para viviendas e incluso la entrada de remesas son elementos considerados como detonantes del aumento de consumo de cemento.

\section{CONCLUSIONES}

En general, los principales resultados de los modelos indican que la tasa de crecimiento de la producción de cemento en México no se explica por elementos de orden interno a la industria, como sería el cambio en el empleo o la 
inversión fija, sino que responde positivamente a la demanda de exportaciones y negativamente a la participación de competidores extranjeros. Dado que las exportaciones aún representan una baja proporción del valor total de la producción, el efecto cuantitativo sobre la tasa de crecimiento del cemento es bajo. Lo mismo sucede con la competencia extranjera. Sin embargo, es evidente la relación negativa con la producción de cemento por lo que consideramos que existen pocas posibilidades de que la industria vea favorable la entrada de mayores volúmenes de importaciones. Considerando que se trata de una estructura de mercado oligopólica este resultado sugiere, de manera indirecta, que la industria cementera tiene mayores incentivos para mantener acuerdos de fijación de precios o cantidades a fin de evitar la entrada de competidores y aumentar los beneficios.

Cuando incorporamos una variable para medir el efecto de la actividad económica nacional, se encontró una fuerte relación causa-efecto entre la producción física de la economía y la tasa de cambio de la producción cementera. Si consideramos que el principal destino del cemento es el mercado nacional, entonces se refuerza la noción de que la industria cementera está fuertemente vinculada al comportamiento de la economía nacional.

Finalmente, reconociendo que las estimaciones de los modelos pueden ser sensibles a errores de especificación, los resultados muestran consistentemente que la mayor participación de competencia externa en México impacta de manera negativa al crecimiento de la industria. Esto confirma el hecho de que la producción nacional de cemento tiene al mercado interno como su principal fuente de crecimiento y fundamenta la estructura oligopólica de las empresas que favorecen la coordinación de la toma de decisiones.

\section{BIBLIOGRAFÍA}

Aguilar, Roberto (2014), "Lafarge-Holcim a la caza de Cemex en su territorio", Alto Nivel, 18 de agosto (consultado el 17 de febrero de 2016), disponible en <http://www.altonivel.com.mx/44689-lafargeholcim-a-lacaza-de-cemex-en-su-territorio.html>

Avalos, Marcos y Claudia Schatan (2003), Condiciones de competencia en el contexto internacional: cemento, azúcar y fertilizantes en Centroamérica, Working paper EGAP-2004-08, Monterrey, México (consultado el 3 de marzo de 2007), disponible en <http://alejandria.ccm.itesm.mx/egap/documentos/EGAP-2004-08.pdf> 
Banco de México, Estadísticas, varios años (consultado el 26 de febrero de 2016), disponible en <http://www.banxico.org.mx/estadisticas/index.html> Barragán Villareal, Juan y Mario Cerutti (2003), Cemex: del mercado interno a la empresa global, Associação Brasileirá de Pesquisadores em História Econômica, V Congresso Brasileiro de História Econômica e $6^{\mathrm{a}}$ Conferência internacional de História de Empresas, Caxambu/2003 (consultado en agosto de 2006), disponible en <http://www.abphe.org.br/arquivos/2003_ juan_ignacio_barragan_mario_cerutti_cemex-del-mercado-interno-a-laempresa-global.pdf>

Blair, Roger y David L. Kaserman (1983), Law and Economics of Vertical Integration and Control, New York, Academic Press.

Blum, Ulrich (2007), "The East German Cement Cartel: Cartel Efficiency and Policy after Economic Transformation", Eastern European Economics, vol. 45, núm. 6, pp. 5-28.

Cámara Nacional del Cemento (Canacem) (2015), Estadísticas, México, CANACEM (consultado el 22 de septiembre de 2015), disponible en $<\mathrm{http}: / /$ www.canacem.org.mx/2006>

Carlton, Dennis (1979), "Vertical Integration in Competitive Markets under Uncertainty", Journal of Industrial Economics, vol. 27, núm. 3, pp. 189209.

Cemex (2016), Tipos de cemento, productos y servicios: cemento (consultado el 26 de febrero de 2016), disponible en < http://www.cemex.com/ES/ ProductosServicios/TiposCemento.aspx>

Cohen-Meidan, Maya (2013), "The Heterogeneous Effects of Trade Protection: a Study of US Antidumping Duties on Portland Cement", Review of Industrial Organization, vol. 42, pp. 369-394.

Cook, Robert (1994), "Cement Exports from Mexico", TED Case Studies, vol. 3, núm. 2.

Corrales, Salvador (2006), Redes productivas en la Industria Acerera. El caso de Altos Hornos de México, S. A. 1982-2002, Tijuana, El Colegio de la Frontera Norte.

De la Garza, Óscar y Julio Arteaga García (2011), “Análisis de la competencia en la Industria Cementera en México”, EconoQuantum, vol. 8, núm. 1, pp. 73-89.

De la Rosa, Tomás (2016), "El cemento más caro del mundo" (consultado el 4 de mayo de 2016), disponible en <http://www.economiahoy.mx/economiahoy/opinion/noticias/6632952/04/15/El-cemento-mas-caro-delmundo.html> 
Dickey, David A. y Wayne A. Fuller (1981), "Likelihood Ratio Statistics for Autoregressive Time Series with a Unit Root”, Econometrica, vol. 49, núm. 4, pp. 1057-1072.

Engle, Robert F. and Granger C. W. J. (1987), "Co-Integration and Error Correction: Representation, Estimation, and Testing", Econometrica, vol. 55, núm. 2, pp. 251-276.

García, Eduardo (2004), "Cemex y su escaramuza con Mary Nour reaviva la polémica sobre la competencia en la industria", Revista Sentido Común (consultado en agosto de 2006), disponible en <http://www.sentidocomun.com.mx/artículo_buscado.phtml>

Ghemawat, Pankaj y Catherine Thomas (2005), Multinational Agglomeration in the Cement Industry: Patterns, Drivers, and Performance Implications, Harvard Business School (consultado en febrero de 2006), disponible en <www.rotman.utoronto.cal_baum/workshop/Ghemawat_worksho.pdf>

Gutiérrez, Salvador y Francisco Núnéz (2002), Caso Trabajo Final: Cemex, construyendo el futuro, México, ITESo (consultado en agosto de 2006), disponible en $<$ http://iteso. $\mathrm{mx} /$ fnunez/eegutierrez/final.doc $>$

Hernández, Ma. Vianney (2004), Caso de Estudio: Cemex, México, iteso (consultado en agosto de 2006), disponible en <http://iteso.mx/-fnunez/ ee2004hernandezcemex.doc>

Hernández Pérez, David (2004), Gaceta Parlamentaria, Exposición de Motivos para regular los precios del cemento en el mercado nacional e internacional, Gaceta LIX/1SPR-162388, 18 de agosto, México, Senado de la República (consultado en agosto de 2006), disponible en <http://www.senado.gob. $\mathrm{mx} /$ index.php?ver=sp\&mn=2\&sm=2\&id=2388>

Instituto Nacional de Estadística y Geografía (INEGI) (2015a), Encuesta Anual de la Industria Manufacturera. Varios años, México (consultado el 16 de febrero de 2016), disponible en <http://www.inegi.org.mx/sistemas/bie/?i dserPadre $=10800070 \#$ D10800070>

(2015b), Encuesta Mensual de la Industria Manufacturera. Varios años, México (consultado el 16 de febrero de 2016), disponible en <http://www. inegi.org.mx/sistemas/bie/?idserPadre=10800070\#D10800070>

(2015c), Encuesta Industrial Mensual Ampliada. Varios años, México (consultado el 11 de marzo de 2016), disponible en <http://www.inegi. org.mx/sistemas/bie/?idserPadre=10800070\#D10800070>

Johansen, S and K. Juselius (1990), "Maximum Likelihood Estimation and Inference on Co-integration with Application for the Demand for Money", Oxford Bulletin of Economics and Statistics, vol. 52, núm. 2, pp. 169210 . 
Kang, N. and K. Sakai (2000), "International Strategic Alliances: Their Role in Industrial Globalization”, OECD Science, Technology and Industry Working Papers, 2000/5, OECD

Klein, A. y H. Palsson (1996), "Is There Anticompetitive Behavior in the Central Canadian Cement Industry? Testing Arbitrage Cost Hypothesis", Canadian Journal of Economics, pp. 343-356.

Kumakan, Gabesh y Saúl Martínez (2008), "Evolución reciente de la industria de cemento: un estudio comparativo entre México y la India”, Revista Mexicana de Estudios sobre la Cuenca del Pacifico, vol. 2, núm. 3, pp. 165-202.

Levenstein, Margaret y Valerie Suslow (2006), "What Determines Cartel Success?", Journal of Economic Literature, vol. 44, núm. 1, pp. 43-95.

Phillips, Peter C. B. y Pierre Perron (1988), "Testing for a Unit Root in Time Series Regression”, Biometrika, vol. 75, núm. 2, pp. 335-346.

Pozas, María de los Ángeles (1999), Mexican Firms in the New Global Economy, PhD, Dissertation, The John Hopkins University, Baltimore, Maryland, USA.

Salomón, Alfredo (2006), "La Industria del Cemento en México", Comercio Exterior, vol. 56, núm. 9, pp. 812-824.

Secretaría de Economía (2006), Acuerdo México-Estados Unidos sobre Comercio de Cemento. Dirección de Comunicación Social (consultado en agosto de 2006), disponible en <http://www.economia-nci.gob.mx/sphp_pages/ sala_prensa/textobd.php?res=943>

(2016), Sistema de Información Arancelaria vía Internet SIAVI (consultado el 15 de marzo de 2016), disponible en <http://www.economia-snci. gob.mx>

Shantayanan, Deverajan y Dani Rodrik (1989), "Trade Liberalization in Developing Countries: do Imperfect Competition and Scale Economies Matter?”, The American Economic Review, vol. 79, núm. 2, pp. 283-287.

Sterner, Thomas (1990), "Energy Efficiency and Capital Embodied Technical Change: The Case of Mexican Cement Manufacturing", The Energy Journal, vol. 11, núm. 2, pp. 155-167.

Torres, Enrique (2014), "El nuevo competidor en la Industria Cementera", Forbes, 2 de octubre (consultado el 24 de febrero de 2016), disponible en <http://www.forbes.com.mx/el-nuevo-competidor-en-la-industria-cementera>

Torres, Armando (2004), "Disputa por cemento ruso", Revista Fortuna, año II, núm. 20 (consultado en agosto de 2006), disponible en <http://revistafortuna.com.mx/opciones/archivo/2004/septiembre/html/negocios/ disputa.htm> 
US Department of Commerce (2009), Gray Portland Cement and Clinker from Mexico: Initiation of Changed Circumstances Review, Preliminary Results of Review, International Trade Administration, Federal Register, vol.74, núm. 30, pp. 7393-7394 (consultado el 26 de febrero de 2016), disponible en <http://www.strtrade.com/media/publication/333_2009February-16-ita_mexico_cement.pdf>

Varian, Hal R. (2011), Microeconomía intermedia, $8^{\text {a }}$ ed, Antoni Bosch, Barcelona.

Vera Martínez, Paola (2013), "La industria del cemento entre la sustentabilidad y la inestabilidad financiera" [tesis de doctorado], unam, Programa de Posgrado en Ciencias de la Administración, diciembre (consultado el 24 de febrero de 2016), disponible en <http://132.248.9.195/ptd2013/ noviembre/0705167/0705167.pdf> 